\title{
Inhibition of Lactate Removal by Ketone Bodies in Rat Liver

\author{
Evidence for a Quantitatively Important Role of the Plasma Membrane \\ Lactate Transporter in Lactate Metabolism
}

\author{
Hilary K. Metcalfe, John P. Monson, Simon G. Welch, and Robert D. Cohen \\ Medical Unit and Department of Biochemistry, The London Hospital Medical College, University of London, London, United Kingdom
}

\begin{abstract}
We studied the effect of DL-3-hydroxybutyrate and acetoacetate on lactate transport into isolated hepatocytes and on lactate removal in the isolated perfused rat liver. Ketone bodies inhibited lactate transport into isolated hepatocytes (maximum, 35\% at concentrations of 10-20 mM). Lactate removal and glucose production by perfused livers were examined before and after the introduction of a constant infusion of hydroxybutyrate, acetoacetate, or appropriate control into the portal venous limb. Lactate removal was significantly inhibited within $10 \mathrm{~s}$ of the appearance of increasing concentrations of ketone bodies in the effluent. Corresponding decreases in glucose production were observed. The dependence of inhibition on D-3-hydroxybutyrate concentration was documented in isolated perfused livers (maximum inhibition of lactate removal, $58 \%$ at $14 \mathrm{mM}$ ). This phenomenon could be a factor in the development of lactic acidosis accompanying ketoacidosis, and indicates that plasma membrane lactate transport may determine the rate of hepatic lactate removal.
\end{abstract}

\section{Introduction}

We have previously described a stereoselective, $\mathrm{pH}$ dependent, high affinity carrier mechanism for lactate entry into isolated rat hepatocytes (1) and these findings have been confirmed by Fafournoux et al. (2). We have postulated that this mechanism might be rate-limiting for overall lactate removal under certain circumstances in intact liver $(1,3)$. Recent studies have shown that lactate binds stereoselectively and in a saturable fashion to a protein of 26,000 molecular weight ( $\mathrm{mol} \mathrm{wt}$ ) in preparations of rat and human hepatocyte plasma membranes (4). We suggest that this binding protein may be the transporter or a fragment of it.

The hepatocyte plasma membrane lactate transporter, like monocarboxylate transporters in other tissues, is probably a semispecific process and is inhibited by pyruvate and 2-oxoglutarate (4). The theoretical possibility thus arises that lactate transport and overall hepatic lactate uptake might be inhibited in vivo under circumstances in which high concentrations of a competing monocarboxylate occur. Because 3-hydroxybutyrate

Portions of this work were presented at the Medical Research Society Meeting, Oxford, United Kingdom, 1984; and at the Fourth European Symposium on Metabolism, Padua, Italy, May 1985.

Received for publication 19 December 1985 and in revised form 22 April 1986.

J. Clin. Invest.

(c) The American Society for Clinical Investigation, Inc.

0021-9738/86/09/0743/05 $\$ 1.00$

Volume 78, September 1986, 743-747 and lactate have been shown to share the same plasma membrane carrier mechanism in several tissues $(5,6)$, we were interested in the possible interrelationships between transport of these monocarboxylates in hepatocytes, especially in view of the fact that clinical ketoacidosis is frequently accompanied by lactic acid accumulation.

We report here the effect of 3-hydroxybutyrate and acetoacetate on lactate transport into isolated hepatocytes. In addition, we have shown immediate inhibition by 3-hydroxybutyrate of lactate removal by isolated perfused livers and shown that lactate transport may be rate-limiting for hepatic lactate removal.

\section{Methods}

Materials. Sodium D-hydroxybutyrate was kindly provided by Dr. D. H. Williamson and was found by enzymatic analysis to contain $93 \%$ of the D-isomer. Sodium DL-3-hydroxybutyrate was obtained from BDH Chemicals, Ltd, Poole, United Kingdom. Sodium [U $\left.-{ }^{14} \mathrm{C}\right] \mathrm{L}-\mathrm{lactate}$, tritiated water, and hydroxy $\left[{ }^{14} \mathrm{C}\right]$ methyl inulin (specific radioactivities, 161 $\mathrm{mCi} / \mathrm{mmol}, 100 \mathrm{mCi} / \mathrm{ml}$, and $17.1 \mathrm{mCi} / \mathrm{mmol}$, respectively) were obtained from Amersham International, Bucks, United Kingdom; bovine serum albumin (BSA) (fraction V) was obtained from Miles Laboratories, Slough, United Kingdom; and $\mathrm{L}+$ )-lactic acid and lithium acetoacetate were obtained from Sigma Chemical Co., Poole, Dorset, United Kingdom. These and other chemicals were of the highest grade available commercially.

Lactate transport studies. Isolated rat hepatocytes were prepared from male Sprague Dawley rats starved for $48 \mathrm{~h}$ (weight, 200-250 g) as previously described (1). The effect of DL-3-hydroxybutyrate and acetoacetate on the entry of $\left[{ }^{14} \mathrm{C}\right] \mathrm{L}-$ lactate into hepatocytes in $15 \mathrm{~s}$ was examined using a silicone oil separation procedure (1). Briefly, aliquots of hepatocyte suspension (wet wt, $30-60 \mathrm{mg} / \mathrm{ml}$ ) in Krebs bicarbonate buffer (7) were preincubated for $20 \mathrm{~min}$ before the simultaneous addition of $0.25 \mu \mathrm{Ci}$ $\left[{ }^{14} \mathrm{C}\right] \mathrm{L}$-lactate and a range of concentrations of either sodium DL-3-hydroxybutyrate or lithium acetoacetate. Lithium acetoacetate was used because of its ready availability in the solid form. Similar concentrations of sodium or lithium chloride were used in parallel experiments as a control for possible osmotic or cationic effects on lactate entry. After 15 $s$ the incubations were terminated by rapid centrifugation of the cells through silicone oil (specific gravity, 1.04) into $1.0 \mathrm{M}$ perchloric acid. ${ }^{3} \mathrm{H}_{2} \mathrm{O}$ was used as a cell water marker. The calculation method of intracellular activity with a correction for extracellular contamination was as previously described (1). We have shown that at $15 \mathrm{~s}<12 \%$ of counts have reached metabolites beyond lactate (1).

Rates of glucose formation were determined by incubating aliquots of cell suspension in the presence of $1 \mathrm{mM}$ lactate with or without 20 mM sodium DL-3-hydroxybutyrate. The incubations were terminated after $20 \mathrm{~min}$ by the addition of $0.6 \mathrm{M}$ perchloric acid and glucose concentrations measured in the acid extract by the method of Stein (8).

Isolated perfused liver studies. Livers from male Sprague Dawley rats starved for $48 \mathrm{~h}$ (weight, 150-200 g) were perfused using the constant flow technique described by Cohen et al. (9). The medium consisted of Krebs bicarbonate buffer (7), BSA ( 20 g/liter) predialysed against Krebs buffer for $48 \mathrm{~h}$, and washed expired human erythrocytes (17\% packed cell volume). The perfusion medium was gassed in a reservoir oxygenator at $37^{\circ} \mathrm{C}$, with a mixture of $\mathrm{O}_{2} / \mathrm{CO}_{2}(95: 5 \%)$ to achieve an initial $\mathrm{PCO}_{2}$ of $\sim 5.0 \mathrm{kPa}$ and a $\mathrm{pH}$ of 7.4 . Flow rate was $7.5 \mathrm{ml} / \mathrm{min}$ per $100 \mathrm{~g}$ rat 
weight. A change in perfusate $\mathrm{pH}$ was achieved by lowering the bicarbonate content and replacing it with chloride. After an initial 20-min recirculating period, the livers were perfused in a nonrecirculating mode with frequent portal and hepatic venous sampling for metabolite analyses. The initial lactate concentration in the perfusate was $1.18 \pm 0.09$ $\mathrm{mM}(n=20)$, which is below the apparent Michaelis constant $\left(K_{\mathrm{m}}\right)(1.9$ $\mathrm{mM}$ [1]) for the lactate transporter. After a further $4 \mathrm{~min}$, a constant infusion of either sodium DL-3-hydroxybutyrate, sodium D-3-hydroxybutyrate, or lithium acetoacetate was started at a point in the circuit close to the insertion of the portal cannula, to achieve a portal perfusate concentration of 10-20 mM. In separate control experiments similar concentrations of saline or lithium chloride were infused. Portal and hepatic venous sampling was continued for a further $6 \mathrm{~min}$.

When intracellular D-hydroxybutyrate was to be determined, $22 \mu \mathrm{Ci}$ ${ }^{3} \mathrm{H}_{2} \mathrm{O}$ and $7.5 \mu \mathrm{Ci}$ hydroxy $\left[{ }^{14} \mathrm{C}\right]$ methyl inulin were added to the perfusate at the start of the perfusion for subsequent determination of the volumes of intracellular and extracellular compartments (10). $1 \mathrm{~min}$ after the start of the D-hydroxybutyrate infusion, the pedicle of the left lobe of the liver was ligated, the lobe was excised and immediately freeze-clamped in tongs cooled in liquid nitrogen. Simultaneously, perfusate flow rate was diminished by $30 \%$, i.e., in proportion to the weight of liver removed (10). 5 min after the start of the infusion, the right lobe was freezeclamped.

$\mathrm{pH}, \mathrm{PCO}_{2}$, and $\mathrm{PO}_{2}$ in the portal and hepatic venous perfusate were measured using an analyzer (Micro 413; Instrumentation Laboratory, Harrington, United Kingdom). For metabolite measurements, 2-ml samples of perfusate were added to $4 \mathrm{ml} 0.6 \mathrm{M}$ perchloric acid and stored at $-20^{\circ} \mathrm{C}$ until analysis. Lactate and glucose were measured by the methods of Hohorst (11) and Stein (8). Lactate removal and glucose production were calculated using the Fick principle. Acetoacetate and D-3-hydroxybutyrate were measured in perchlorate extracts of perfusate and powdered freeze-clamped liver by the procedure of Williamson et al. (12). In experiments where DL-3-hydroxybutyrate was infused, total perfusate ketone concentration was derived from measured acetoacetate and D-hydroxybutyrate using the previously determined ratio of $D$ to $L$ isomer (1:1) in the racemic mixture.

Results are expressed as means \pm SEM unless otherwise indicated. Significance between mean values was analyzed using two-tailed unpaired $t$ tests.

\section{Results}

Effect of 3-hydroxybutyrate and acetoacetate on $\left[{ }^{14} \mathrm{C}\right] L$-lactate entry into isolated hepatocytes. The results from these experiments are shown in Figs. 1 and 2. Lactate entry into isolated hepatocytes was inhibited by both DL-3-hydroxybutyrate and acetoacetate in a concentration-dependent manner. Inhibition reached a plateau of $\sim 35 \%$, at 3-hydroxybutyrate and acetoacetate concentrations of $10-20 \mathrm{mM}$, after subtraction of values for control experiments using sodium or lithium chloride. Rates of glucose production from lactate $(1 \mathrm{mM})$ by isolated hepatocytes were unaffected by incubation with $20 \mathrm{mM}$ DL-3-hydroxybutyrate for $20 \mathrm{~min}$ (data not shown), thus indicating that 3-hydroxybutyrate does not inhibit intracellular lactate metabolism. One would not expect glucose production in the isolated cell model to be decreased by inhibition of plasma membrane lactate transport because of the component of entry by passive diffusion, which is artefactually increased and is sufficient in isolated cells to render the transport component non-rate-limiting (1).

Effect of ketone bodies on lactate removal by the isolated perfused liver. A representative example of the results from this series of experiments is shown in Fig. 3. Overall lactate removal was studied for $10 \mathrm{~min}$, with the first $\mathbf{4} \mathrm{min}$ used as a control period. After 4 min a constant portal ketone infusion was started, in this case sodium DL-3-hydroxybutyrate, calculated to give a hepatic portal perfusate concentration of $\sim 20 \mathrm{mM}$. Within 10

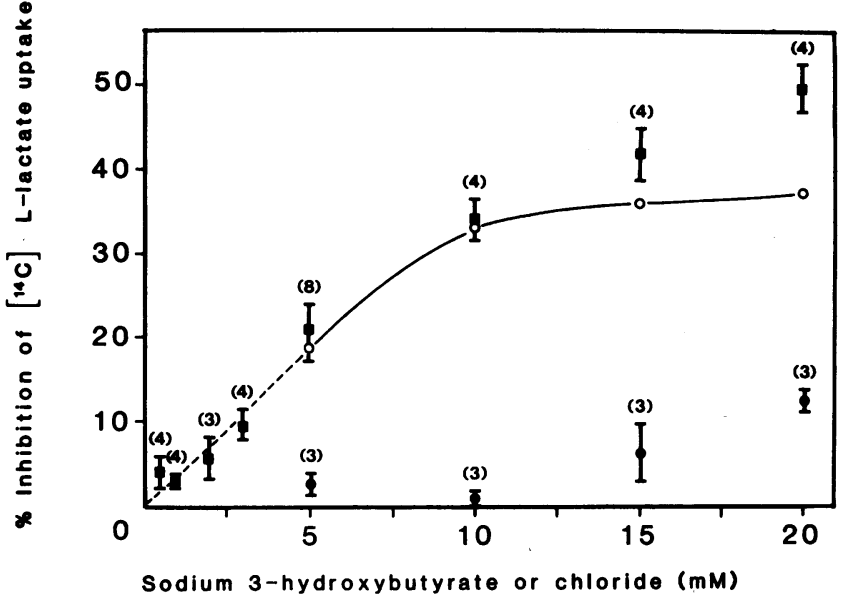

Figure 1. The effect of sodium DL-3-hydroxybutyrate and sodium chloride on the entry of $\left[{ }^{14} \mathrm{C}\right] \mathrm{L}$-lactate into isolated rat hepatocytes. $\mathrm{Al}$ iquots of hepatocyte suspension were preincubated for $20 \mathrm{~min}$ before the addition of $0.25 \mu \mathrm{Ci}$ of $\left[{ }^{14} \mathrm{C}\right] \mathrm{L}$-lactate with or without sodium DL-3-hydroxybutyrate or sodium chloride. $15 \mathrm{~s}$ after the addition cells were centrifuged into perchloric acid as described under Methods. The solid line and open symbols represent the difference between the sodium hydroxybutyrate ( $\bullet$ ) and sodium chloride effects (๑). Results are means \pm SEM with number of observations in parentheses. Mean $\left[{ }^{14} \mathrm{C}\right]$ lactate entry in the absence of sodium DL-3-hydroxybutyrate or sodium chloride was $1,959 \pm 193 \mathrm{dpm} / \mu \mathrm{l}$ cell water $(n=4)$.

$s$ of the appearance of increasing concentrations of ketones in the hepatic venous effluent, a decrease in lactate removal rate was observed and this effect persisted for at least the 6 min studied. The inhibition of lactate removal reached a maximum of $24 \%$, which remained constant until the end of the experiment. Inhibition of lactate removal was followed by a decrease in glu-

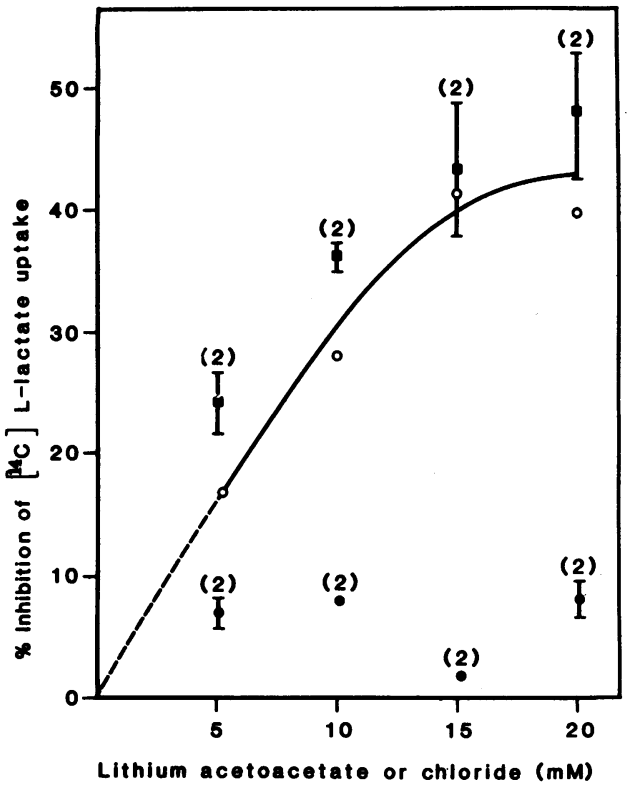

Figure 2. The effect of lithium acetoacetate and lithium chloride on the entry of $\left[{ }^{14} \mathrm{C}\right] \mathrm{L}$-lactate into isolated rat hepatocytes. Experiments were performed as described under Methods. The effect of lithium acetoacetate ( $\square$ ) and lithium chloride ( $(\bullet)$ were studied. The solid line and open symbols represent the net effect of acetoacetate. Each point represents the mean and range of two experiments. Mean $\left[{ }^{14} \mathrm{C}\right]$ lactate entry in the absence of additions was $3,035 \mathrm{dpm} \pm 609 \mathrm{dpm} / \mu \mathrm{l}$ cell water $(n=4)$. 


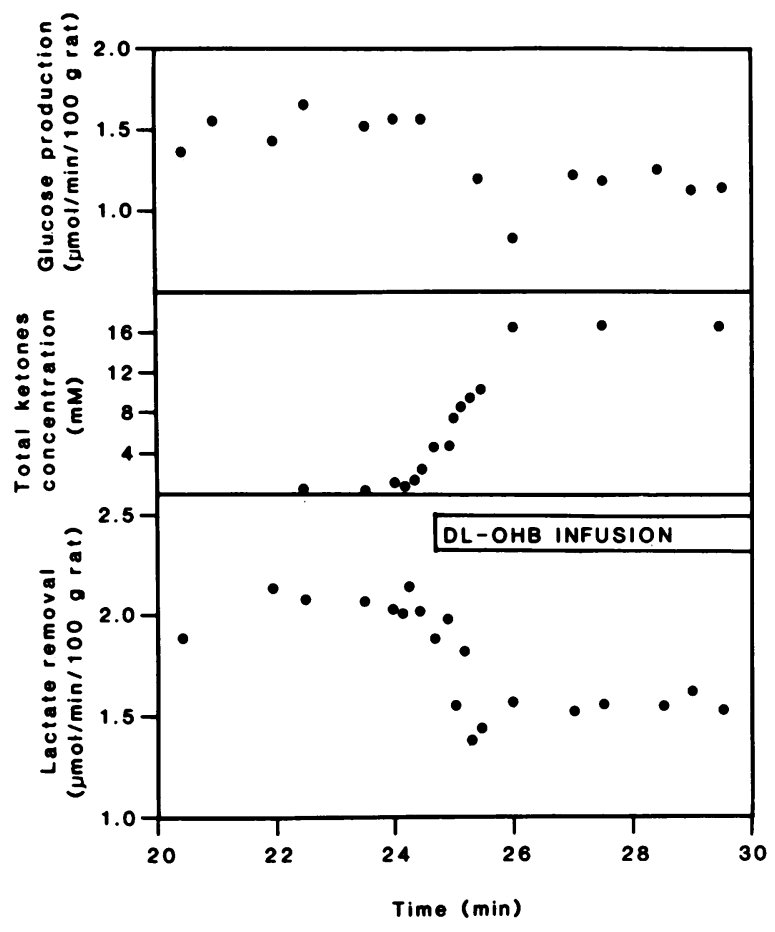

Figure 3. The effect of sodium DL-3-hydroxybutyrate on lactate removal and glucose production in the isolated perfused rat liver. The liver was perfused using the constant flow technique and in the nonrecirculating mode as described under Methods. The perfusate lactate concentration was $1.2 \mathrm{mM}$. A constant infusion of sodium DL-3-OHB was introduced at the time shown into the portal line to achieve a portal perfusate concentration of $15-20 \mathrm{mM}$. Data from a representative perfusion are shown.

cose production. Fig. 4 illustrates a control perfusion; no decrease in lactate removal was observed when a similar concentration of sodium chloride was infused. Cessation of hydroxybutyrate infusion resulted in a rapid restoration of preinfusion lactate removal rates (Fig. 5). These results are summarized in Table I. Mean inhibition of lactate removal $(-23.6 \%)$ was highly significantly different from that observed during sodium chloride infusion $(+6.1 \%)$.

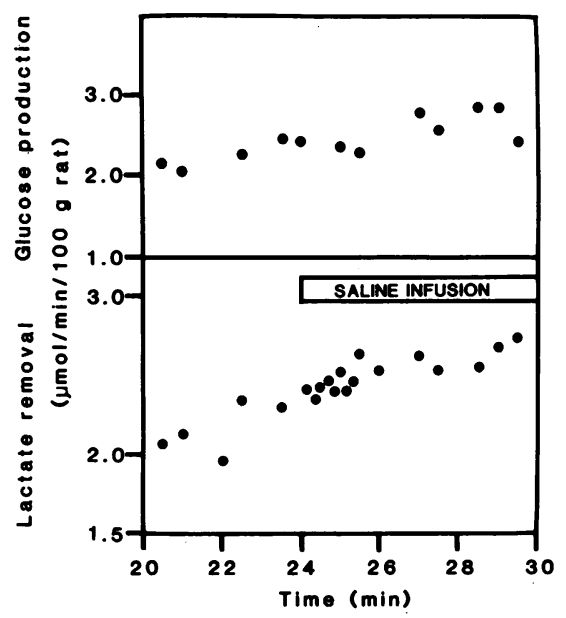

Figure 4. The effect of sodium chloride on lactate removal and glucose production in the isolated perfused rat liver. A constant infusion of sodium chloride was introduced into the portal line to achieve a perfusate concentration of $\sim 20 \mathrm{mM}$. Data from a representative perfusion are shown.

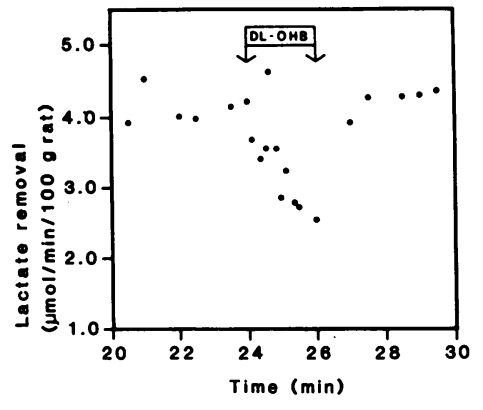

Figure 5. Reversibility of the effect of sodium DL-3hydroxybutyrate on lactate removal in the isolated perfused rat liver. A constant infusion of sodium DL-3-hydroxybutyrate was delivered into the portal line during the interval indicated by the arrows to achieve a maximum portal perfusate concentration of $\sim 10 \mathrm{mM}$ DL-hydroxybutyrate.
Similar results were obtained with infusions of acetoacetate (Table I) and the naturally occurring D-isomer of 3-hydroxybutyrate. Fig. 6 shows the relationship between inhibition of lactate removal and the concentration of D-hydroxybutyrate infused. Maximum inhibition ( $46 \%)$ is achieved at concentrations of $12 \mathrm{mM}$ and above. In a single experiment in which perfusate $\mathrm{pH}$ was lowered to 6.8 , infusion of DL-hydroxybutyrate inhibited lactate removal by $37 \%$ (i.e., comparable with results at normal $\mathrm{pH}$ ).

In one experiment, intracellular hydroxybutyrate was measured as described in Methods at 1 and 5 min after the start of D-hydroxybutyrate infusion. The concentration of D-hydroxybutyrate in cell and perfusate water, together with the inhibition of lactate removal achieved, are shown in Fig. 7. At $1 \mathrm{~min}$ the intracellular concentration had already reached $9.27 \mathrm{mM}$; the perfusate concentration at this time was probably artefactually low, due to difficulties in precise timing of tissue and perfusate samples at a time when perfusate hydroxybutyrate concentration was rapidly rising. By $5 \mathrm{~min}$ the cell water and perfusate concentration were comparable, in the region of $20 \mathrm{mM}$.

Table I. Effect of Sodium DL-3-hydroxybutyrate, Lithium Acetoacetate, and Appropriate Controls on Lactate Removal and Glucose Production in the Isolated Perfused Rat Liver

\begin{tabular}{lll}
\hline Infusion & $\begin{array}{l}\text { Percent change in } \\
\text { lactate removal }\end{array}$ & $\begin{array}{l}\text { Percent change in } \\
\text { glucose production }\end{array}$ \\
\hline $20 \mathrm{mM} \mathrm{NaCl}$ & $\begin{array}{l}+6.1 \pm 5.7 \\
(n=6)\end{array}$ & $\begin{array}{l}+18.1 \pm 6.4 \\
(n=6)\end{array}$ \\
$20 \mathrm{mM}$ DL-OHB & $\begin{array}{l}-23.6 \pm 6.8^{*} \\
(n=7)\end{array}$ & $-19.1 \pm 4.2 \ddagger$ \\
& $+10.9 \pm 0.2$ & $(n=5)$ \\
$20 \mathrm{M} \mathrm{LiCl}$ & $(n=2)$ & - \\
& $-18.9 \pm 7.6$ & - \\
$20 \mathrm{mM} \mathrm{LiAcAc}$ & $(n=2)$ & -52 \\
& -37.0 & \\
(acidotic liver) & &
\end{tabular}

Percent change in lactate removal and glucose production rates by isolated perfused rat livers after commencement of the portal venous infusions indicated. Mean rates of lactate removal and glucose production for the preinfusion period were $3.02 \pm 0.27$, SEM $(n=26)$ and $2.82 \pm 0.37(n=12) \mu \mathrm{mol} / \mathrm{min}^{-1}$ per $100 \mathrm{~g}$ rat, respectively.

OHB, 3-hydroxybutyrate; LiAcAc, lithium acetoacetate.

$* P<0.005$.

$\ddagger P<0.001$ 


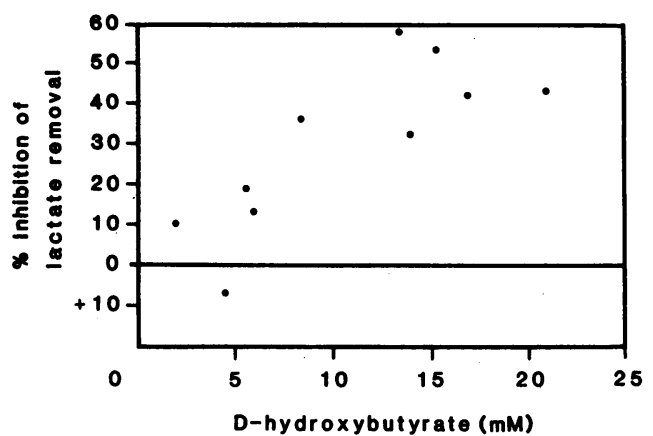

Figure 6. Concentration-dependent effect of sodium D-3-hydroxybutyrate on lactate removal in the isolated perfused rat liver. Percent change in lactate removal after delivery of a constant infusion of D-3hydroxybutyrate into the portal line to achieve the perfusate concentrations indicated. Points represent individual perfused liver preparations.

\section{Discussion}

The present studies show a dose-dependent inhibition by DL-hydroxybutyrate of lactate entry into isolated rat hepatocytes. The possibility that the effect of hydroxybutyrate on lactate entry was in some way related to changes in intracellular redox state was made unlikely by the observation that acetoacetate inhibits lactate transport to a similar extent. The degree of inhibition of lactate transport by hydroxybutyrate and acetoacetate is incomplete, with a maximum of $45 \%$ inhibition being observed. This finding is comparable with our previous observation of incomplete saturation of lactate entry by increasing concentration of lactate and incomplete inhibition of entry by $\alpha$-cyano-3-hydroxycinnamate, pyruvate, and 2-oxoglutarate $(1,4)$. Kinetic analysis of the lactate entry mechanism $(1,3)$ suggests that these observations may be explained by a two-component system with a high affinity saturable carrier and a second component that does not saturate with concentrations up to $28 \mathrm{mM}$ and that is not subject to inhibition. We have suggested (13) that the nonsaturable component is passive nonionic diffusion, which may be artefactually increased in the isolated cell model because of the larger available surface area. We have recently shown that

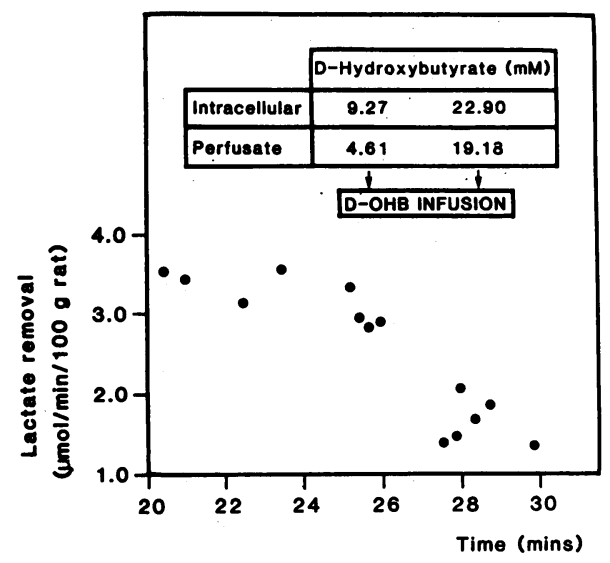

Figure 7. Intracellular and perfusate concentrations of D-hydroxybutyrate and effect on lactate removal during infusion of D-hydroxybutyrate. The arrows indicate the times at which liver lobes were sampled as described in the text; the boxes indicate the hydroxybutyrate concentrations corresponding to the arrows. hepatic plasma membrane D-3-hydroxybutyrate transit is carriermediated and inhibited by lactate (14). It is thus possible that these monocarboxylates share the same carrier; alternatively, hydroxybutyrate may inhibit the lactate carrier, but not utilize it as a transport mechanism.

In previous studies (1) of lactate transport into isolated rat hepatocytes it was not possible to draw firm conclusions regarding the quantitative importance of the lactate transporter in overall lactate metabolism although it has been suggested that under certain circumstances it might become rate-limiting in vivo for overall lactate removal (1-3). The inhibition of lactate removal by 3-hydroxybutyrate in the perfused liver experiments described in this paper provides the first indication that inhibition of the transporter may limit lactate uptake and metabolism in intact liver.

The data indicate that lactate removal is inhibited within 10 s of exposure of the liver to 3-hydroxybutyrate. We have shown (1) that $15 \mathrm{~s}$ after addition of $\left[{ }^{14} \mathrm{C}\right] \mathrm{L}$-lactate to hepatocytes, $<12 \%$ of counts have reached metabolites distal to lactate, and the studies of Fafournoux and colleagues (2) further support this conclusion. There is no reason to suppose that the situation is markedly different in intact perfused liver, in which the overall mean transit time of lactate to glucose is $75 \mathrm{~s}(15)$. We therefore conclude that the inhibition of lactate removal by hydroxybutyrate is due to an effect on lactate transport into the cell rather than on subsequent steps of lactate metabolism. The similar inhibitory effect achieved with acetoacetate again makes redox changes an unlikely explanation. The possibility that cell acidification due to inward carriage of protons as undissociated hydroxybutyric acid (23) was responsible for a net decrease in lactate entry by non-ionic diffusion is made unlikely by our observation (unpublished) that cell pH (measured by $\left[{ }^{14} \mathrm{C}\right]$ dimethyloxazolidine-dione distribution) is unchanged $6 \mathrm{~min}$ after the start of infusion. Nor would such a mechanism account for the saturation of the hydroxybutyrate effect. The effect of 3-hydroxybutyrate was rapidly reversible and, importantly, is demonstrated by $D$-hydroxybutyrate, the natural isomer. These findings could have important implications for the control of hepatic gluconeogenesis and pathogenesis of lactic acidosis. Perfusions performed at pH 6.8 did not increase the inhibitory effect of hydroxybutyrate and thus any enhancement of lactate transport that might have been expected as a consequence of acidosis (1, 2) appears to be quantitatively unimportant under these conditions.

An inhibitory effect of ketones on lactate transport would account for the observation of Shaw et al. (16) of inhibition of gluconeogenesis by ketones in the perfused dog liver and also for the hypoglycaemic effects of injections of high doses of acetoacetate in rats (17). This is in contrast to the findings of other groups who observed stimulation of gluconeogenesis from lactate by ketones, in the perfused rat liver $(18,19)$. Contradictory findings in different species have been attributed to differences in the distribution of the gluconeogenic enzymes, particularly phosphoenol pyruvate carboxykinase, between the cytosolic and mitochondrial compartments (20). In the rat this enzyme is virtually confined to the cytosol while in other species a significant portion is located in the mitochondria. Apparently contrasting results within species require further explanation. Patel et al. (18) attributed the observed stimulation of gluconeogenesis to increased entry of pyruvate into the mitochondrial compartment on the monocarboxylate translocator. However, the findings of Patel et al. (18) and Arinze et al. (19) are not incompatible with 
the present study as different lactate and ketone concentrations were employed. In the studies of Patel et al. (18) which used 20 mM lactate, a concentration far exceeding the apparent $K_{\mathrm{m}}$ for the plasma membrane lactate transporter (1.9 mM [1]), it is possible that sufficient lactate could have entered liver cells by passive diffusion to saturate the gluconeogenic pathway, despite inhibition of plasma membrane lactate transport. We have confirmed that in the presence of high perfusate lactate concentrations (6-20 mM) lactate removal and glucose production are not inhibited by D-hydroxybutyrate (data not shown). In contrast, in the present study employing physiological lactate concentrations $(\sim 1 \mathrm{mM})$, we suggest that a large proportion of the lactate entry into the liver occurs via the plasma membrane lactate transporter; changes in lactate uptake due to inhibition of the transporter by 3-hydroxybutyrate were therefore readily observable. At the low concentration of hydroxybutyrate used by Arinze et al. (19) we observed little or no effect; the higher concentrations at which we observed clear inhibition of lactate uptake nevertheless remained well within the range seen in diabetic ketoacidosis.

Considering the quantitative importance of hepatic lactate removal in overall lactate disposal (21), it is significant that high concentrations of ketones may inhibit hepatic lactate metabolism. Thus, clinical conditions in which plasma ketone concentrations approach those employed in the present experiments might be associated with inhibition of hepatic lactate removal. This could partly explain the frequently observed lactic acid accumulation in diabetic ketoacidosis, which has previously been attributed (21) to increased peripheral glycolysis due to reduced tissue perfusion. It could be argued that infusion of hydroxybutyrate might not have the same effect on net lactate entry as might occur in diabetic ketoacidosis, in which ketone bodies arise within the cell. Thus, if the lactate transporter is symmetrical, high intracellular concentrations of ketones might inhibit efflux of lactate, and the net effect on lactate uptake might be opposite to that observed by us. However, the data in Fig. 7 indicate that the intracellular concentration of hydroxybutyrate rises very rapidly after the start of the infusion to levels comparable with that of the perfusate. Despite these high intracellular concentrations, which are comparable to those observed in livers from severely diabetic ketoacidotic rats (mean tissue concentra-

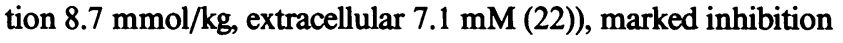
of lactate removal persists. These observations suggest that the lactate transporter may not be symmetrical and that the effects observed in the present study may be clinically relevant. Nevertheless, lactate accumulation is only present in a proportion of patients with ketoacidosis of various origins. Whether lactate accumulation does occur may depend on the relative magnitude in individual patients of $(a)$ suppression of peripheral glycolysis by acidosis, $(b)$ adequacy of peripheral perfusion, $(c)$ stimulation of lactate transport into liver by acidosis $(1,2)$, and $(d)$ the effect of ketone bodies described here.

Our findings clearly indicate that inhibition of hepatic plasma membrane lactate transport results in reduced overall hepatic lactate removal. This hitherto neglected site of control of lactate metabolism should be considered, in addition to intracellular events, in future studies of controlling factors in acid-base homeostasis and gluconeogenesis. The observations also suggest that the hydroxybutyrate-inhibitable portion of lactate removal can be used as an indicator of lactate-transporter activity in studies of physiological and pathological variations of lactate uptake in perfused rat liver.

\section{Acknowledgment}

This work was supported by a grant from the Medical Research Council (United Kingdom) and The London Hospital Special Trustees.

\section{References}

1. Monson, J. P., J. A. Smith, R. D. Cohen, and R. A. Iles. 1982. Evidence for a lactate transporter in the plasma membranes of rat hepatocytes. Clin. Sci. 62:411-420.

2. Fafournoux, P., C. Demigne, and C. Remesy. 1985. Carrier-mediated uptake of lactate in rat hepatocytes. J. Biol. Chem. 260:292-299.

3. Monson, J. P. 1983. M.D. thesis. University of London, United Kingdom. 1-169.

4. Welch, S. G., H. K. Metcalfe, J. P. Monson, R. D. Cohen, R. M. Henderson, and R. A. Iles. 1984. L(+)-Lactate binding to preparations of rat hepatocyte plasma membranes. J. Biol. Chem. 259:15264-15271.

5. Andersen, B. L., H. L. Tarpley, and D. M. Regan. 1978. Characterization of $\beta$-hydroxybutyrate transport in rat erythrocytes and thymocytes. Biochim. Biophys. Acta. 508:525-538.

6. Oldendorf, W. H. 1973. Carrier-mediated blood-brain barrier transport of short-chain monocarboxylic organic acids. Am. J. Physiol. 224:1450-1453.

7. Krebs, H. A., and K. Henseleit. 1932. Untersuchungen über die Harnstoffbildung im Tierkörper. Hoppe-Seyler's Z. Physiol. Chem. 210: 33-36.

8. Stein, M. W. 1983. In Methods of Enzymatic Analysis. H. U. Bergmeyer, editor. Academic Press, Inc., New York. 117-123.

9. Cohen, R. D., R. A. Iles, and M. H. Lloyd. 1973. Liver (rat). In Isolated organ perfusion. H. D. Ritchie and J. D. Hardcastle, editors. Crosby, Lockwood and Staples, London. 120-134.

10. Cohen, R. D., R. A. Iles, D. Barnett, M. E. O. Howell, and J. Strunin. 1971. The effect of changes in lactate uptake on the intracellular pH of the perfused rat liver. Clin. Sci. 41:159-170.

11. Hohorst, H. J. 1962. In Methods of Enzymatic Analysis. H. U. Bergmeyer, editor. Academic Press, Inc., New York. 266-270.

12. Williamson, D. H., J. Mellanby, and H. A. Krebs. 1962. Enzymatic determination of $\mathrm{D}(-) \mathrm{B}-$ hydroxybutyic acid and acetoacetic acid in blood. Biochem. J. 82:90-98.

13. Cohen, R. D., R. M. Henderson, R. A. Iles, J. P. Monson, and J. A. Smith. 1982. CIBA Symposium on Metabolic Acidosis. Pitman Books, Ltd., London. 20-35.

14. Metcalfe, H. K., J. P. Monson, S. G. Welch, and R. D. Cohen. 1985. The mechanism of 3-hydroxybutyrate efflux in isolated rat hepatocytes. Eur. J. Clin. Invest. 15:A8.

15. Exton, J. H., and C. R. Park. 1967. Control of gluconeogenesis in liver. General features of gluconeogenesis in the perfused liver of rats. J. Biol. Chem. 242:2622-2636.

16. Shaw, J. H. F., and R. R. Wolfe. 1984. Glucose production in the perfused dog liver: effect of free fatty acids and ketones. J. Surg. Res. 37:437-442.

17. Tidwell, H. C., and H. E. Axelrod. 1948. Blood sugar after injection of acetoacetate. J. Biol. Chem. 172:179-184.

18. Patel, T. B., L. L. Barron, and M. S. Olson. 1984. The stimulation of hepatic gluconeogenesis by acetoacetate precursors. J. Biol. Chem. 259:7523-7531.

19. Arinze, I. J., A. J. Garber, and R. W. Hanson. 1973. The regulation of gluconeogenesis in mammalian liver. J. Biol. Chem. 248:2266-2274.

20. Hanson, R. W. 1974. The choice of animal species for studies of metabolic regulation. Nutr. Rev. 32:1-8.

21. Cohen, R. D., and H. F. Woods. 1983. Lactic acidosis revisited. Diabetes. 32:181-191.

22. Blackshear, P. J., P. A. H. Holloway, and K. G. M. M. Alberti. 1975. The effects of inhibition of gluconeogenesis on ketogenesis in starved and diabetic rats. Biochem. J. 148:353-62.

23. Sies, H., G. Noack, and K.-H. Halder. 1973. Carbon dioxide concentration and the distribution of monocarboxylate and $\mathrm{H}^{+}$ions between intracellular and extracellular spaces of haemoglobin-free perfused rat liver. Eur. J. Biochem. 38:247-258. 